\title{
First-order rotation solution of an oblate rigid body under the torque of a perturber in circular orbit
}

\author{
M. Lara, T. Fukushima, and S. Ferrer \\ 1 Real Observatorio de la Armada, 11110 San Fernando, Spain \\ e-mail:mlara@roa.es \\ 2 National Astronomical Observatory, Mitaka, 181-8588 Tokyo, Japan \\ 3 Departamento de Matemática Aplicada, Universidad de Murcia, 30100 Murcia, Spain
}

Received 15 December 2009 / Accepted 17 May 2010

\section{ABSTRACT}

\begin{abstract}
Aims. We discuss the rotational motion of an axisymmetric rigid body under the torque exerted by a massive point orbiting in a circular Keplerian orbit. The perturbation of the torque-free motion is modelled by applying MacCullagh's approximation.

Methods. Through successive approximations we use the unperturbed motion solution to get a first order solution for the Hamilton equations, which we apply to the complete problem.

Results. The solution of the perturbed problem is computed in closed form without the usual series expansions, and shows the main effects on the dynamics of the rigid body that are introduced by the perturbation. Remarkably, special configurations are discovered, for which the perturbation only introduces periodic oscillations in the torque-free motion. An application to the dwarf-planet Ceres shows the reliability of the analytical solution.
\end{abstract}

Key words. celestial mechanics - methods: analytical - minor planets, asteroids: individual: Ceres

\section{Introduction}

The description of the rotational motion of celestial bodies may require high precision theories as for the rotation of the Earth (Souchay et al. 1999; Shirai \& Fukushima 2000; Getino \& Ferrandiz 2001; Mathews et al. 2002; Escapa et al. 2002) or the physical librations of the Moon (Moons 1982). However, as high-precision measurements are not yet available for other celestial bodies, and given the uncertainties on several physical parameters, the rotation may be described with simpler theories. It is commonly assumed that the rotating body is rigid and that the perturbing body is a mass point or an oblate body (Henrard 2005). The consideration of simple nonrigid models may show however that new frequencies may appear in the motion, which can introduce long period contributions to the rigid case (Barkin \& Ferrándiz 2005).

Rotational theories for specific bodies include numerical values of physical parameters. On the contrary, generic rotational motion theories aim to retain frequencies and amplitudes as formal functions of the parameters like, for instance, the moments of inertia of the perturbed body. Therefore, the models used should be definitely simpler, so they can provide the required insight on the dynamics (Kinoshita 1972). We aim to follow this way.

Perturbations of the rotational state of celestial bodies of the solar system produced by direct or indirect effects due to the gravitational attraction of other bodies are normally small compared with their torque-free evolution. That is why the rotation of solar system bodies is commonly studied only up to first order effects in the perturbation. In addition, in many cases the rotation occurs very close to the principal axis of inertia, which justifies performing series expansions of the quantities involved in the study (Kinoshita 1972, 1977). Here we proceed differently, and find a first order solution to the perturbed rigid body motion that avoids series expansions.

We study the rotation of an oblate spheroid perturbed by a massive distant point. The assumption of axisymmetry is very close to the real situation of many bodies of the solar system. Besides, it has a zero order, torque-free motion solution that is free from elliptic integrals. We consider a perturbing body moving in a circular orbit, and refer the attitude of the rigid body to the inertial plane where the orbit lies. Referring the solution to a different inertial plane requires only performing the rotations defined by the inclination of the orbital plane and its argument of the node. With these simplifying assumptions of axisymmetry and a perturber on a circular orbit, the arguments of the solution depend on frequencies that only involve the orbital mean motion and the torque-free rotation rate, and reveal the main characteristics of the perturbed motion.

The rotational dynamics of celestial bodies has a long history associated to the different models considered. Among them is the d'Alambert problem, a planet modelled by a oblate ellipsoid revolving on a Keplerian orbit of small eccentricity around a fixed star, under the only influence of gravitational forces. It still challenges mathematical studies of long-term dynamics and stability, and it is only recently that partial results have been obtained for some conditions of the parameters involved (see Chierchia \& Gallavotti 1994; Mastropietro 2001, and references therein).

Apart from the astronomical interest in theories related to the long-term proper and precessional motions, the roto-translatory dynamics of artificial satellites has renewed the interest in this field since the beginning of the space era. New models were proposed, including other perturbations (the third body in 
particular), and the new research included the search for special solutions related to relative equilibria (Lagrangian and Eulerian points) and their stability. Without aiming at reviewing the vast literature on the topic, we just mention the pioneering work of Belestkii (see Beletskii 1966, and references therein), soon after followed by Chernous'ko (1963), and others (Holland \& Sperling 1969; Kinoshita 1972; Barkin 1980; Markeev 1985; Bois 1986, and others). At present the roto-translatory dynamics of binaries systems is another area of research, in which classical models do require a full revision, because the orbit can no longer be taken as a Keplerian ellipse (see Scheeres 2009, and references therein).

Of special relevance to the present research is the work of Crenshaw \& Fitzpatrick (1968) who, apparently unaware of previous works of astronomers, compute a Hamilton-Jacobi solution to the torque-free motion that introduces the Andoyer angles (Andoyer 1923) as auxiliary variables in the derivation procedure. They finally find these auxiliary angles to be a suitable set of variables for describing the perturbed motion when combined with Euler-type inclination angles and the energy. For the particular case of a non-precessing orbital plane, their secular solution matches the secular terms of ours.

To compute our theory we used Andoyer variables and Hamiltonian formalism (Andoyer 1923; Deprit 1967). The zero order of the Hamiltonian is the torque-free motion, and the perturbation is considered in the MacCullagh's approximation. The Hamiltonian of the torque-free motion in Andoyer variables is Liouville-integrable in the axisymmetric case. It depends only on two momenta, and consequently shows evidence of the linear motion of the two conjugate angles. This zero order solution is introduced on the right side of the Hamilton equations of the perturbed problem and integrated by quadrature. Constructing our theory this way introduces resonant terms in the solution that, specifically, prevent its application to the spin-orbit 1:1 resonance. Dealing with resonant cases requires the use of specific variables (Bouquillon et al. 2003) and is out of the scope of the present paper.

Despite the rough assumptions of our model it may fit the dwarf-planet Ceres, whose shape is well described by an oblate spheroid to the limit of measurement, or to a lesser extent to the asteroid 16 Psyche or even to 4 Vesta (Thomas et al. 2005; Drummond \& Christou 2008; Carry et al. 2008), all of them orbiting the sun with relatively low eccentricities. Besides, the perturbation of the torque-free motion is of the order of $10^{-7}$ for Ceres and 4 Vesta - targets of actual interest since the approval of the NASA Dawn mission (Russell et al. 2003) - and less than that for 16 Psyche. Therefore, the first order approach seems to be justified.

The application of our theory to Ceres reveals secular variations in all angles at a precessional rate of a few radians per Julian century. Periodic oscillations appear in all variables, the most relevant amplitudes are related to Ceres' semiannual effects except for the modulus of the angular momentum, for which only higher order, low-period effects related to the free rotation of Ceres are apparent. Because of the symmetries of our model, the solution is free from the usual middle-term effects associated to the rigid body polar motion.

\section{Unperturbed motion in Andoyer's variables: axisymmetric case}

The kinetic energy of rotation of a rigid body about its centre of mass is $T=\frac{1}{2}\left(A \omega_{1}^{2}+B \omega_{2}^{2}+C \omega_{3}^{2}\right)$, where $A \leq B \leq C$ denote the principal moment of inertia and $\left(\omega_{1}, \omega_{2}, \omega_{3}\right)$ are the components of the instantaneous rotation vector in the frame of the principal axis of the body. Alternatively, it may be expressed as $T=\frac{1}{2}\left(a_{1} g_{1}^{2}+a_{2} g_{2}^{2}+a_{3} g_{3}^{2}\right)$, where $a_{1}=A^{-1} \geq a_{2}=B^{-1} \geq a_{3}=$ $C^{-1}$, and $\left(g_{1}, g_{2}, g_{3}\right)$ are the components of the angular momentum vector in the body frame. The angular momentum vector is preserved in the inertial frame for the torque-free motion and, therefore, its modulus $G=\sqrt{g_{1}^{2}+g_{2}^{2}+g_{3}^{2}}$ is constant.

The Hamiltonian of the torque-free rotation is obtained from the kinetic energy by expressing either the angular velocity or the angular momentum in a convenient set of canonical variables like, for instance, Euler angles and their canonical extension. Specifically, the Hamiltonian is reduced to one degree of freedom when using Andoyer variables $(\lambda, \mu, \nu, \Lambda, M, N)$ that link the body and inertial frames through an intermediate frame with the $z$-axis defined by the direction of the angular momentum vector, the $x$-axis defined by the intersection of the plane perpendicular to the angular momentum vector and the inertial $x-y$ plane, and the $y$ axis defining a direct frame (Andoyer 1923). Then the position of the intermediate plane with respect to the inertial frame is defined by its argument of the node $\lambda$ and inclination $I=\arccos (\Lambda / M)$, where $\Lambda$ is the projection of the angular momentum vector on the $z$-axis of the inertial frame, and $M=G$. The position of the equatorial plane of the rigid body with respect to the intermediate plane is defined by its argument of the node $\mu$, and inclination $J=\arccos (N / M)$, where $N$ is the projection of the angular momentum vector over the $z$-axis of the body frame. Finally, the $x$-axis of the body frame is located on the equatorial plane of the body through the angle $v$.

The transformation from Euler to Andoyer variables is canonical (Deprit 1967), and the Hamiltonian of the torque-free motion is

$\mathcal{H}=\frac{1}{2}\left(a_{1} \sin ^{2} v+a_{2} \cos ^{2} v\right)\left(M^{2}-N^{2}\right)+\frac{1}{2} a_{3} N^{2}$,

where $\lambda, \Lambda$ and $\mu$ are cyclic and therefore $\lambda=\lambda_{0}, \Lambda=\Lambda_{0}$, and $M=M_{0}$ are constant. The integration of the Hamilton equations for $\mu, v$, and $N$ can be solved by quadrature, and accepts a closed form solution in elliptic integrals.

For those bodies with axisymmetry with respect to the axis of maximum inertia $a_{1}=a_{2}$, a reasonable approximation to many celestial bodies, and the Hamiltonian reduces to

$\mathcal{H}=\frac{1}{2} a_{1} M^{2}-\frac{1}{2}\left(a_{1}-a_{3}\right) N^{2}$,

of trivial integration. Thus,

$\lambda=\lambda_{0}, \quad \Lambda=\Lambda_{0}, \quad M=M_{0}, \quad N=N_{0}$,

are constant, and the rotation angles evolve linearly with time

$\mu=\mu_{0}+a_{1} M_{0} t, \quad v=v_{0}-\left(a_{1}-a_{3}\right) N_{0} t$.

\section{Perturbed motion: first order approach}

Now we consider the perturbed motion of the oblate rigid body above, of mass $m$, under the gravitational pull of distant point of mass $m_{1}$. The Hamiltonian is $\mathcal{H}=H_{0}+H_{1}$, where $H_{0}$ is the zero order Hamiltonian given by Eq. (2) and the perturbing term $H_{1}$ is taken in MacCullag's (1840) approximation

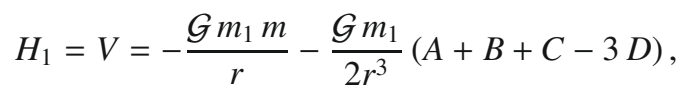


M. Lara et al.: Rotation of an oblate rigid body under external torque

where $D=A \alpha^{2}+B \beta^{2}+C \gamma^{2}$ is the moment of inertia of the rigid body with respect to an axis in the direction of the line joining its centre of mass with the perturber, of direction cosines $\alpha, \beta$, and $\gamma\left(\alpha^{2}+\beta^{2}+\gamma^{2}=1\right), \mathcal{G}$ is the gravitational constant, and $r$ is the distance between the centres of mass of the perturbing and perturbed bodies. Note that we may drop the Keplerian term from the Hamiltonian, because it does not produce any effect on the rotation.

If we assume that the perturbing body is orbiting in a circular Keplerian orbit of a radius $r$, we find for the axisymmetric case

$H_{1}=\epsilon\left(1-3 \gamma^{2}\right)$,

with

$\epsilon=-\frac{G m_{1}}{2 r^{3}}\left(\frac{1}{a_{3}}-\frac{1}{a_{1}}\right)$.

Besides, if we further require that the perturbing body's orbit lies in the $x-y$ plane of the inertial frame, the orbital reference frame is related to the body frame through the direction cosines by means of the rotations

$\left(\begin{array}{c}\alpha \\ \beta \\ \gamma\end{array}\right)=R_{3}(-v) R_{1}(-J) R_{3}(-\mu) R_{1}(-I) R_{3}(\vartheta)\left(\begin{array}{l}1 \\ 0 \\ 0\end{array}\right)$,

where $\vartheta=\theta-\lambda$ and $\theta$ is the usual polar coordinate of the orbital motion, which evolves linearly with time for the circular motion $\theta=n t$. As usual $R_{i}(\phi)$ means a rotation of amplitude $\phi$ about the $i$-axis, $i=1,2,3$, where 1 is used for $x, 2$ for $y$ and 3 for $z$.

Then

$\gamma=\sin J \cos \vartheta \sin \mu-(\cos J \sin I+\sin J \cos I \cos \mu) \sin \vartheta$,

and

$$
\begin{aligned}
\frac{H_{1}}{\epsilon}= & \frac{1}{4}\left(1-3 \cos ^{2} J\right)\left(1-3 \cos ^{2} I-3 \sin ^{2} I \cos 2 \vartheta\right) \\
& -\frac{3}{4} \sin I \sin 2 J[2 \cos I \cos \mu \\
& -(1+\cos I) \cos (2 \vartheta-\mu)+(1-\cos I) \cos (2 \vartheta+\mu)] \\
& +\frac{3}{8} \sin ^{2} J\left[2 \sin ^{2} I \cos 2 \mu+(1+\cos I)^{2} \cos (2 \vartheta-2 \mu)\right. \\
& \left.+(1-\cos I)^{2} \cos (2 \vartheta+2 \mu)\right] .
\end{aligned}
$$

Because of the symmetries of our model, the angle $v$ is cyclic in the time-dependent Hamiltonian (7), and therefore the projection of the angular momentum on the $z$-axis of the body frame remains constant in the perturbed problem $N=N_{0}$.

The equations of motion of the perturbed problem are obtained from Hamilton equations

$\frac{\mathrm{d}(\lambda, \mu, v)}{\mathrm{d} t}=\frac{\partial \mathcal{H}}{\partial(\Lambda, M, N)}, \quad \frac{\mathrm{d}(\Lambda, M, N)}{\mathrm{d} t}=-\frac{\partial \mathcal{H}}{\partial(\lambda, \mu, \nu)}$.

Then

$$
\begin{aligned}
& \frac{\mathrm{d} M}{\mathrm{~d} t}=-\frac{3 \epsilon}{4 M^{4}} \sum_{i=0}^{2} \sum_{j=-2}^{2} M_{i, j}^{*} \sin (i \vartheta+j \mu) \\
& \frac{\mathrm{d} \Lambda}{\mathrm{d} t}=-\frac{3 \epsilon}{4 M^{4}} \sum_{i=0}^{2} \sum_{j=-2}^{2} \Lambda_{i, j}^{*} \sin (2 \vartheta+j \mu)
\end{aligned}
$$

$$
\begin{aligned}
\frac{\mathrm{d} v}{\mathrm{~d} t}= & -\left(a_{1}-a_{3}\right) N-\frac{3 \epsilon}{2 M} \frac{N}{M}\left(1-3 \frac{\Lambda^{2}}{M^{2}}\right) \\
& +\frac{3 \epsilon}{4 M^{4}} N \sum_{i=0}^{2} \sum_{j=-2}^{2} v_{i, j}^{*} \cos (i \vartheta+j \mu) \\
\frac{\mathrm{d} \lambda}{\mathrm{d} t}= & -\frac{3 \epsilon}{2 M} \frac{\Lambda}{M}\left(1-3 \frac{N^{2}}{M^{2}}\right) \\
& +\frac{3 \epsilon}{4 M^{4}} \sum_{i=0}^{2} \sum_{j=-2}^{2} \lambda_{i, j}^{*} \cos (i \vartheta+j \mu) \\
\frac{\mathrm{d} \mu}{\mathrm{d} t}= & a_{1} M+\frac{3 \epsilon}{2 M}\left[\left(1-6 \frac{N^{2}}{M^{2}}\right) \frac{\Lambda^{2}}{M^{2}}+\frac{N^{2}}{M^{2}}\right] \\
& +\frac{3 \epsilon}{2 M^{5}} \sum_{i=0}^{2} \sum_{j=-2}^{2} \mu_{i, j}^{*} \cos (i \vartheta+j \mu),
\end{aligned}
$$

where $M_{i, j}^{*}, \Lambda_{i, j}^{*}, \mu_{i, j}^{*}, v_{i, j}^{*}$ and $\lambda_{i, j}^{*}$ are coefficients of the momenta, whose values are given in Table 1.

\subsection{First order solution}

The perturbed problem is likely non-integrable, but assuming that the perturbation is small, we try a first order solution in the small parameter $\epsilon$ by the straightforward method of successive approximations as follows.

First we replace Andoyer variables into the right side of Hamilton equations, Eqs. (8)-(11), by its the zero order solution. Thus, for initial conditions $\left(\mu_{0}, v_{0}, \lambda_{0}, M_{0}, N_{0}, \Lambda_{0}\right)$, we introduce the first order solution Eqs. (3) and (4) into the right side of Eqs. (8)-(12). Then, Eqs. (8)-(12) only depend on time and can be trivially integrated by quadratures to give

$$
\begin{aligned}
M= & M_{1}-\frac{3}{4} \epsilon \sum_{i=0}^{2} \sum_{j=-2}^{2} \frac{M_{i, j}}{i n+j a_{1} M} \cos (i \varphi+j f) \\
\Lambda= & \Lambda_{1}-\frac{3}{4} \epsilon \sum_{i=0}^{2} \sum_{j=-2}^{2} \frac{\Lambda_{i, j}}{i n+j a_{1} M} \cos (i \varphi+j f) \\
v= & v_{1}-\left(a_{1}-a_{3}\right) N t-\frac{3 \epsilon}{2 M}\left[\left(1-3 \cos ^{2} I\right) \cos J\right] t \\
& -\frac{3 \epsilon}{4 M} \sum_{i=0}^{2} \sum_{j=-2}^{2} \frac{v_{i, j}}{i n+j a_{1} M} \sin (i \varphi+j f) \\
\lambda= & \lambda_{1}-\frac{3 \epsilon}{2 M}\left[\left(1-3 \cos ^{2} J\right) \cos I\right] t \\
& -\frac{3 \epsilon}{4 M} \sum_{i=0}^{2} \sum_{j=-2}^{2} \frac{\lambda_{i, j}}{i n+j a_{1} M} \sin (i \varphi+j f) \\
\mu= & \mu_{1}+a_{1} M t+\frac{3 \epsilon}{2 M}\left[\cos ^{2} J+\left(1-6 \cos ^{2} J\right) \cos ^{2} I\right] t \\
& -\frac{3 \epsilon}{4 M} \sum_{i=0}^{2} \sum_{j=-2}^{2} \frac{\mu_{i, j}}{i n+j a_{1} M} \sin (i \varphi+j f),
\end{aligned}
$$

where $M_{i, j}, \Lambda_{i, j}, v_{i, j}, \lambda_{i, j}$, and $\mu_{i, j}$ are coefficients of the momenta, whose values are given in Tables 2 and 3 , and we call

$f=a_{1} M t+\mu_{0}, \quad \varphi=n t-\lambda_{0}$,

and, in the right side of the equations, $M=M_{0}, N=N_{0}$, $\Lambda=\Lambda_{0}, I=\arccos \left(\Lambda_{0} / M_{0}\right), J=\arccos \left(N_{0} / M_{0}\right)$. Note that 
Table 1. Non-zero values of the coefficients in Eqs. (8)-(12).

\begin{tabular}{cccc}
\hline \hline$(i, j)$ & $M_{i, j}^{*}$ & $\Lambda_{i, j}^{*}$ & $v_{i, j}^{*}$ \\
\hline$(2,-2)$ & $(\Lambda+M)^{2}\left(M^{2}-N^{2}\right)$ & $(\Lambda+M)^{2}\left(M^{2}-N^{2}\right)$ & $-(M+\Lambda)^{2}$ \\
$(2,-1)$ & $2 N(M+\Lambda) \sqrt{M^{2}-\Lambda^{2}} \sqrt{M^{2}-N^{2}}$ & $4 N(M+\Lambda) \sqrt{M^{2}-\Lambda^{2}} \sqrt{M^{2}-N^{2}}$ & $-2 \frac{M+\Lambda}{N} \frac{\sqrt{M^{2}-\Lambda^{2}}}{\sqrt{M^{2}-N^{2}}}\left(2 N^{2}-M^{2}\right)$ \\
$(2,0)$ & - & $-2\left(M^{2}-\Lambda^{2}\right)\left(M^{2}-3 N^{2}\right)$ & $6\left(M^{2}-\Lambda^{2}\right)$ \\
$(2,1)$ & $2 N(M-\Lambda) \sqrt{M^{2}-\Lambda^{2}} \sqrt{M^{2}-N^{2}}$ & $-4 N(M-\Lambda) \sqrt{M^{2}-\Lambda^{2}} \sqrt{M^{2}-N^{2}}$ & $2 \frac{M-\Lambda}{N} \frac{\sqrt{M^{2}-\Lambda^{2}}}{\sqrt{M^{2}-N^{2}}}\left(2 N^{2}-M^{2}\right)$ \\
$(2,2)$ & $-(M-\Lambda)^{2}\left(M^{2}-N^{2}\right)$ & $(M-\Lambda)^{2}\left(M^{2}-N^{2}\right)$ & $-(M-\Lambda)^{2}$ \\
$(0,2)$ & $-2\left(M^{2}-\Lambda^{2}\right)\left(M^{2}-N^{2}\right)$ & - & $-2\left(M^{2}-\Lambda^{2}\right)$ \\
$(0,1)$ & $4 N \Lambda \sqrt{M^{2}-\Lambda^{2}} \sqrt{M^{2}-N^{2}}$ & - & $4 \frac{\Lambda}{N} \frac{\sqrt{M^{2}-\Lambda^{2}}}{\sqrt{M^{2}-N^{2}}}\left(2 N^{2}-M^{2}\right)$ \\
\hline
\end{tabular}

\begin{tabular}{ccc}
\hline$(i, j)$ & $\lambda_{i, j}^{*}$ & $\mu_{i, j}^{*}$ \\
\hline$(2,-2)$ & $(M+\Lambda)\left(M^{2}-N^{2}\right)$ & $(M+\Lambda)\left[M N^{2}-\Lambda\left(M^{2}-2 N^{2}\right)\right]$ \\
$(2,-1)$ & $2 N \frac{\sqrt{M^{2}-N^{2}}}{\sqrt{M^{2}-\Lambda^{2}}}\left(M^{2}-2 \Lambda^{2}-M \Lambda\right)$ & $-2 N \sqrt{M^{2}-\Lambda^{2}} \sqrt{M^{2}-N^{2}}\left[M+2 \Lambda-\left(\frac{\Lambda^{2}}{M^{2}-\Lambda^{2}}+\frac{N^{2}}{M^{2}-N^{2}}\right)(M+\Lambda)\right]$ \\
$(2,0)$ & $2 \Lambda\left(M^{2}-3 N^{2}\right)$ & $-6 M^{2} N^{2}-2 \Lambda^{2}\left(M^{2}-6 N^{2}\right)$ \\
$(2,1)$ & $2 N \frac{\sqrt{M^{2}-N^{2}}}{\sqrt{M^{2}-\Lambda^{2}}}\left(M^{2}-2 \Lambda^{2}+M \Lambda\right)$ & $2 N \sqrt{M^{2}-\Lambda^{2}} \sqrt{M^{2}-N^{2}}\left[M-2 \Lambda-\left(\frac{\Lambda^{2}}{M^{2}-\Lambda^{2}}+\frac{N^{2}}{M^{2}-N^{2}}\right)(M-\Lambda)\right]$ \\
$(2,2)$ & $-(M-\Lambda)\left(M^{2}-N^{2}\right)$ & $(M-\Lambda)\left[M N^{2}+\left(M^{2}-2 N^{2}\right) \Lambda\right]$ \\
$(0,2)$ & $-2 \Lambda\left(M^{2}-N^{2}\right)$ & $2 M^{2} N^{2}+2 \Lambda^{2}\left(M^{2}-2 N^{2}\right)$ \\
$(0,1)$ & $-4 N \frac{\sqrt{M^{2}-N^{2}}}{\sqrt{M^{2}-\Lambda^{2}}}\left(M^{2}-2 \Lambda^{2}\right)$ & $4 N \Lambda \sqrt{M^{2}-\Lambda^{2}} \sqrt{M^{2}-N^{2}}\left(2-\frac{\Lambda^{2}}{M^{2}-\Lambda^{2}}-\frac{N^{2}}{M^{2}-N^{2}}\right)$ \\
\hline
\end{tabular}

the integration constants $M_{1}, \Lambda_{1}, \ldots, \lambda_{1}$ may be different from the initial conditions $M_{0}, \Lambda_{0}, \ldots, \lambda_{0}$, respectively, because corresponding perturbation terms in Eqs. (13)-(17) do not necessarily vanish at $t=0$.

We remark, however, that the solution given by Eqs. (13)-(17) and (18) does not include first order terms. A first order solution in $\epsilon$ requires a new approximation. The variables $M, \Lambda, \mu, v, \lambda$, in the right members of Eqs. (8)-(12), are now replaced by the values given by Eqs. (13)-(17) and (18). After the replacement, Eqs. (8)-(12) depend only on time and can be integrated by quadrature.

Except for $\mu$, up to the first order in $\epsilon$ the new solution is formally equal to that of Eqs. (13)-(16), but to avoid the introduction of mixed terms in higher orders that will be produced by a naive series expansion, we hold linear arguments of time of the order of $\epsilon$ in circular functions, in the style of the LindstedtPoincaré approach. Hence, now

$$
\begin{aligned}
& f=a_{1} M t+\frac{3 \epsilon}{2 M}\left[\cos ^{2} J+\left(1-6 \cos ^{2} J\right) \cos ^{2} I\right] t+\mu_{0}, \\
& \varphi=n t+\frac{3 \epsilon}{2 M}\left(1-3 \cos ^{2} J\right) \cos I t-\lambda_{0},
\end{aligned}
$$

and $M=M_{1}, N=N_{0}, \Lambda=\Lambda_{1}$.

With respect to $\mu$, the zero order term $a_{1} M$ in the right side of Eq. (12) causes the new approximation of $M$, Eq. (13), not only to change the zero order frequency $a_{1} M=a_{1} M_{1}$, but also introduces new first order periodic terms in the solution for $\mu$. The frequencies of these first order terms are improved with a new iteration of the procedure, which leaves everything unaltered except for the first order correction to $\mu$ :

$\delta=\frac{3 \epsilon}{4 M} \sum_{i=0}^{2} \sum_{j=-2}^{2} \frac{\delta_{i, j}}{i n+j a_{1} M} \sin (i \varphi+j f)$, to which Eq. (19) applies. Therefore, the first order solution to $\mu$ is obtained by adding Eqs. (20) to (17), using Eq. (19) and $M=M_{1}, N=N_{0}, \Lambda=\Lambda_{1}$.

In this way we obtain a first-order solution, Eqs. (13)-(17) and (19)-(20), of the perturbed problem Eqs. (8)-(12), which has a zero-order part

$$
\begin{aligned}
& \mu=\mu_{1}+a_{1} M_{1} t, \quad M=M_{1}, \quad \lambda=\lambda_{1}, \\
& v=v_{1}-\left(a_{1}-a_{3}\right) N_{0} t, \quad N=N_{0}, \quad \Lambda=\Lambda_{1},
\end{aligned}
$$

that is the torque-free motion of the axisymmetric body for the initial conditions $\left(\mu_{1}, \nu_{1}, \lambda_{1}, M_{1}, N_{0}, \Lambda_{1}\right)$.

Euler angles $\phi, \psi$, and $\theta$, for precession, rotation, and nutation, respectively, are recovered from Andoyer variables with standard identities of spherical trigonometry. Then,

$$
\psi=\lambda+\arctan \left(\frac{\sin \mu \sin J}{\sin I \cos J+\cos I \cos \mu \sin J}\right),
$$

$\theta=\arccos (\cos I \cos J-\sin I \sin J \cos \mu)$,

$\phi=v+\arctan \left(\frac{\sin \mu \sin I}{\sin J \cos I+\cos J \cos \mu \sin I}\right)$,

the series developments of which would produce time explicit expressions of Euler angles up to the first order of $\epsilon$.

Note that, alternatively to the straightforward method of successive approximations, the first order solution of Eqs. (8)-(12) may be computed with the perturbation theory. Specifically, the Lie-series approach (Hori 1966; Deprit 1969; Campbell \& Jefferys 1970) has been profusely used in the literature when dealing with Hamiltonian problems (see Getino \& Ferrándiz 1991; Morbidelli et al. 1994, for instance). 
Table 2. Non-zero values of the coefficients in Eqs. (13)-(16).

\begin{tabular}{ccccc}
\hline \hline$(i, j)$ & $M_{i, j}$ & $\Lambda_{i, j}$ & $v_{i, j}$ & $\lambda_{i, j}$ \\
\hline$(2,-2)$ & $-\sin ^{2} J(1+\cos I)^{2}$ & $-\sin ^{2} J(1+\cos I)^{2}$ & $(1+\cos I)^{2} \cos J$ & $-(1+\cos I) \sin ^{2} J$ \\
$(2,-1)$ & $-\sin I \sin 2 J(1+\cos I)$ & $-2 \sin I \sin 2 J(1+\cos I)$ & $-2(1+\cos I)\left(2 \sin I \sin J-\frac{\sin I}{\sin J}\right)$ & $(\cos I+\cos 2 I) \frac{\sin 2 J}{\sin I}$ \\
$(2,0)$ & - & $\left(2-6 \cos ^{2} J\right) \sin ^{2} I$ & $-6 \sin ^{2} I \cos J$ & $\cos I\left(4-6 \sin ^{2} J\right)$ \\
$(2,1)$ & $-\sin I \sin 2 J(1-\cos I)$ & $2 \sin I \sin 2 J(1-\cos I)$ & $2(1-\cos I)\left(2 \sin I \sin J-\frac{\sin I}{\sin J}\right)$ & $-(\cos I-\cos 2 I) \frac{\sin 2 J}{\sin I}$ \\
$(2,2)$ & $\sin ^{2} J(1-\cos I)^{2}$ & $-\sin ^{2} J(1-\cos I)^{2}$ & $(1-\cos I)^{2} \cos J$ & $(1-\cos I) \sin ^{2} J$ \\
$(0,2)$ & $2 \sin ^{2} I \sin ^{2} J$ & - & $2 \sin ^{2} I \cos J$ & $2 \cos I \sin ^{2} J$ \\
$(0,1)$ & $-\sin 2 I \sin 2 J$ & - & $4 \cos I\left(2 \sin I \sin J-\frac{\sin I}{\sin J}\right)$ & $\left(2-4 \cos ^{2} I\right) \frac{\sin 2 J}{\sin I}$ \\
\hline
\end{tabular}

Table 3. Non-zero values of the coefficients in Eqs. (17) and (20).

\begin{tabular}{ccc}
\hline \hline$(i, j)$ & $\mu_{i, j}$ & $\delta_{i, j} \times\left(i n+j a_{1} M\right) /\left(a_{1} M\right)$ \\
\hline$(2,-2)$ & $-(1+\cos I)\left(\cos ^{2} J+\cos I \cos 2 J\right)$ & $\sin ^{2} J(1+\cos I)^{2}$ \\
$(2,-1)$ & $-2\left[\frac{\sin I}{\sin J}-(\cos I-2 \cos 2 I) \frac{\sin J}{\sin I}\right] \cos J(1+\cos I)$ & $\sin I \sin 2 J(1+\cos I)$ \\
$(2,0)$ & $2\left[3 \cos ^{2} J+\cos ^{2} I\left(1-6 \cos ^{2} J\right)\right]$ & - \\
$(2,1)$ & $2\left[\frac{\sin I}{\sin J}+(\cos I+2 \cos 2 I) \frac{\sin J}{\sin I}\right] \cos J(1-\cos I)$ & $\sin I \sin 2 J(1-\cos I)$ \\
$(2,2)$ & $-(1-\cos I)\left(\cos ^{2} J-\cos I \cos 2 J\right)$ & $-\sin ^{2} J(1-\cos I)^{2}$ \\
$(0,2)$ & $-2\left[\sin ^{2} I \cos ^{2} J+\cos ^{2} I \sin ^{2} J\right]$ & $-2 \sin ^{2} J \sin ^{2} I$ \\
$(0,1)$ & $4 \cos I \cos J\left[\frac{\sin I}{\sin J}+\left(1-4 \sin ^{2} I\right) \frac{\sin J}{\sin I}\right]$ & $\sin 2 I \sin 2 J$ \\
\hline
\end{tabular}

In our case, up to the first order in $\epsilon$, the Lie-series approach gives the secular Hamiltonian $\mathcal{K}=\langle\mathcal{H}\rangle_{\mu, \vartheta}$ in new (prime) variables

$$
\begin{aligned}
\mathcal{K}= & \frac{1}{2} a_{1} M^{\prime 2}-\frac{1}{2}\left(a_{1}-a_{3}\right) N^{\prime 2} \\
& +\frac{\epsilon}{4}\left(1-3 \cos ^{2} J^{\prime}\right)\left(1-3 \cos ^{2} I^{\prime}\right),
\end{aligned}
$$

and the generating function

$$
\begin{aligned}
W= & -\frac{3}{8} \epsilon\left\{\left(2-6 \cos ^{2} J^{\prime}\right) \sin ^{2} I^{\prime} \frac{\sin 2 \vartheta^{\prime}}{2 n}\right. \\
& -2 \sin I^{\prime} \sin 2 J^{\prime}\left[\left(1+\cos I^{\prime}\right) \frac{\sin \left(2 \vartheta^{\prime}-\mu^{\prime}\right)}{2 n-a_{1} M^{\prime}}\right. \\
& \left.-2 \cos I^{\prime} \frac{\sin \mu^{\prime}}{a_{1} M^{\prime}}-\left(1-\cos I^{\prime}\right) \frac{\sin \left(2 \vartheta^{\prime}+\mu^{\prime}\right)}{2 n+a_{1} M^{\prime}}\right] \\
& -\sin ^{2} J^{\prime}\left[\left(1+\cos I^{\prime}\right)^{2} \frac{\sin \left(2 \vartheta^{\prime}-2 \mu^{\prime}\right)}{2 n-2 a_{1} M^{\prime}}\right. \\
& \left.\left.+2 \sin ^{2} I^{\prime} \frac{\sin 2 \mu^{\prime}}{2 a_{1} M^{\prime}}+\left(1-\cos I^{\prime}\right)^{2} \frac{\sin \left(2 \vartheta^{\prime}+2 \mu^{\prime}\right)}{2 n+2 a_{1} M^{\prime}}\right]\right\},
\end{aligned}
$$

where $\vartheta^{\prime}=n t-\lambda^{\prime}$, and functions in "primes" denote functions of the "prime" variables.

The evolution equations

$$
\begin{aligned}
& \mu^{\prime}=\mu_{0}^{\prime}+a_{1} M^{\prime} t+\frac{3 \epsilon}{2 M^{\prime}}\left[\cos ^{2} J^{\prime}+\left(1-6 \cos ^{2} J^{\prime}\right) \cos ^{2} I^{\prime}\right] t \\
& v^{\prime}=v_{0}^{\prime}-\left(a_{1}-a_{3}\right) N^{\prime} t-\frac{3 \epsilon}{2 M^{\prime}} \cos J^{\prime}\left(1-3 \cos ^{2} I^{\prime}\right) t \\
& \lambda^{\prime}=\lambda_{0}^{\prime}-\frac{3 \epsilon}{2 M^{\prime}} \cos I^{\prime}\left(1-3 \cos ^{2} J^{\prime}\right) t
\end{aligned}
$$

with $M^{\prime}=M_{0}^{\prime}, N^{\prime}=N_{0}^{\prime}$ and $\Lambda^{\prime}=\Lambda_{0}^{\prime}$ constant, are obtained after a trivial integration by quadrature of the corresponding Hamilton equations of $\mathcal{K}$. Note that Eq. (23) is the same as Eq. (30) of Crenshaw \& Fitzpatrick (1968) for their particular case of a non-precessing orbital plane, with the equivalence $\left(\mu^{\prime}, v^{\prime}, \lambda^{\prime}, M^{\prime}, I^{\prime}, J^{\prime}\right) \rightarrow\left(\phi_{\mathrm{H}}, \phi^{\prime}, \psi_{\mathrm{H}}, h, \theta_{\mathrm{H}}, \theta^{\prime}\right)$ and $\epsilon=-K / 3$.

Then, the solution of Eqs. (8)-(12) for a given set of initial conditions is computed by applying the transformation

$$
\begin{aligned}
& \mu=\mu^{\prime}+\partial W / \partial M^{\prime} \\
& v=v^{\prime}+\partial W / \partial N^{\prime} \\
& \lambda=\lambda^{\prime}+\partial W / \partial \Lambda^{\prime} \\
& M=M^{\prime}-\partial W / \partial \mu^{\prime} \\
& N=N^{\prime} \\
& \Lambda=\Lambda^{\prime}-\partial W / \partial \lambda^{\prime}
\end{aligned}
$$

to the values obtained from the evolution equations.

It is easily checked that the direct substitution of Eq. (23) onto the right side of Eq. (24) results up to the first order of $\epsilon$ in the successive approximation solution given by Eqs. (13)-(17) and (19)-(20) - which in turn provides a strong test on the reliability of the solution. Therefore, the use of either the successive approximations solution or the first order solution by Lietransforms is fully equivalent for the first order computations of this paper.

\subsection{Notable cases}

From the simple inspection of the first-order solution above, specifically Eqs. (15)-(17), one can note that there is a critical inclination at which the body evolves, on average, as in the free rotation given by Eq. (21). This remarkable result 
Table 4. Physical and orbital parameters of Ceres (http://ssd. jpl. nasa.gov/).

\begin{tabular}{ll}
\hline \hline mass & $9.4 \times 10^{20} \mathrm{~kg}$ \\
equatorial radius & $487.3 \mathrm{~km}$ \\
polar radius & $454.7 \mathrm{~km}$ \\
rotational period & 0.3781 days \\
orbital period & 1680.5 days \\
semimajor axis & $413832.587 \mathrm{~km}$ \\
heliocentric gravitational constant & $1.327 \times 10^{11} \mathrm{~km}^{3} / \mathrm{s}^{2}$ \\
\hline
\end{tabular}

occurs for $\cos ^{2} J_{0}=\cos ^{2} I_{0}=1 / 3$, or $I_{0}=\arccos \left( \pm 3^{-\frac{1}{2}}\right)$ and $J_{0}=\arccos \left( \pm 3^{-\frac{1}{2}}\right)$, giving rise to the four combinations $I_{0}=J_{0}=54.7356^{\circ}, I_{0}=J_{0}=125.2644^{\circ}, I_{0}=54.7356^{\circ}$ and $J_{0}=125.2644^{\circ}$, and $I_{0}=125.2644^{\circ}$ and $J_{0}=54.7356^{\circ}$, where the first order secular effects in Eqs. (15)-(17) vanish, and the zero order solution Eq. (21) of the perturbed problem Eqs. (13)-(17) is only affected by periodic perturbations. This result was previously obtained by Chernous'ko (1963), who studied a similar problem using the Krylov-Bogoliuvov averaging method. Besides, another critical inclination is easily found for $I_{0}=J_{0}=90^{\circ}$.

On the other hand, these critical inclinations may be derived from the evolution equations, Eq. (23), by imposing that there are no secular perturbations to the rigid body motion.

Note that Andoyer variables are singular for zero inclination of the intermediate plane with respect to either the inertial or the equatorial plane of the body, or both. The case $I=0$ is not of major concern, and the virtual singularity $J=0$ may be avoided by using other sets of variables in the literaure (Fukushima 1994). In addition, the theory does not apply to synchronous orbiting and rotating satellites because of the resonant term $2 n-2 a_{1} M_{0}$ in the denominators of Eqs. (13)-(17). A solution valid for spinorbit resonance would require the introduction of specific resonant variables (Bouquillon et al. 2003).

\section{Numerical comparisons: the Ceres case}

Even though the assumptions above made for the computation of the analytical theory may seem very rough, they fit the dwarfplanet Ceres except perhaps for the non-negligible eccentricity of its orbit around the $\operatorname{sun} e=0.08$. Accepted values of Ceres' orbital and physical parameters of interest are summarized in Table 4. Slight modifications os these values are also found in the literature (Michalak 2000; Carry et al. 2008).

Assuming that Ceres is an spheroid of homogeneous density, we compute

$$
\begin{aligned}
& A=\frac{1}{a_{1}}=\frac{1}{5} m\left(a^{2}+c^{2}\right)=8.35121 \times 10^{25} \mathrm{~kg} \mathrm{~km}^{2}, \\
& C=\frac{1}{a_{3}}=\frac{2}{5} m a^{2}=8.92854 \times 10^{25} \mathrm{~kg} \mathrm{~km}^{2},
\end{aligned}
$$

where $m$ is Ceres' mass, and $a$ and $c$ are Ceres' equatorial and polar radius, respectively. In addition, $n=4.32741 \times 10^{-8} \mathrm{~s}$, and $\epsilon=-5.40548 \times 10^{9} \mathrm{~kg} \mathrm{~km}^{2} / \mathrm{s}^{2}$ from Eq. (6).

In addition, we take as the initial value for $I$ the accepted value of the tilt $I_{0}=3 \mathrm{deg}$ (Thomas et al. 2005), and for $J$ we take a speculative value of $J_{0}=10^{-4} \mathrm{rad}(\sim 20 \mathrm{arcsec})$, thus assuming a small difference between the figure and angular momentum axes that with the actual precision of observations is usually assumed to match (Drummond \& Christou 2008).
For the given values of the parameters and initial conditions, the amplitudes of the unperturbed secular terms for $v$ and $\mu$ in Eqs. (15) and (17) are

$$
\begin{aligned}
& a_{1} M=+6.4893 \times 10^{5}, \\
& -\left(a_{1}-a_{3}\right) N=-4.1960 \times 10^{4},
\end{aligned}
$$

radians per century, respectivelly. The amplitudes of the perturbed secular terms for $\nu, \lambda$ and $\mu$ in Eqs. (15)-(17) are

$$
\begin{aligned}
& -\frac{3 \epsilon}{2 M}\left[\left(1-3 \cos ^{2} I\right) \cos J\right]=-2.9678 \times 10^{-3}, \\
& -\frac{3 \epsilon}{2 M}\left[\left(1-3 \cos ^{2} J\right) \cos I\right]=-2.9759 \times 10^{-3}, \\
& \frac{3 \epsilon}{2 M}\left[\cos ^{2} J+\left(1-6 \cos ^{2} J\right) \cos ^{2} I\right]=+5.9396 \times 10^{-3},
\end{aligned}
$$

$\mathrm{rad} /$ century, respectivelly. Finally, Table 5 shows the period of the different angular frequencies involved in the analytical solution of Ceres, Eqs. (13)-(17) and (20), and the amplitudes of the respective coefficients in each variable $M, \Lambda, \mu, v$, and $\lambda$. In this table, "coefficient" means the coefficient

$$
-\frac{3 \epsilon}{4 M} \frac{x_{i, j}}{i n+j a_{1} M}, \quad x \in(\mu-\delta, v, \lambda),
$$

$$
-\frac{3}{4} \epsilon \frac{X_{i, j}}{i n+j a_{1} M}, \quad X \in(M, \Lambda),
$$

of corresponding periodic terms, where $x_{i, j}$ and $X_{i, j}$ are given in Tables 2 and 3. Note that $\delta$ terms are negligible for Ceres.

As it is apparent from Table 5, the periodic terms with the frequencies $2 a_{1} M$ and $2 n+2 a_{1} M$ are negligible. Besides, the angular frequency $2 n$ is the unique non-negligeble in the solution for $\lambda$. Therefore, for the specific case of Ceres, Eqs. (13)-(17) and (20) can be further simplified.

Now we check that the frequencies predicted by the theory are apparent from the direct numerical integration of the equations of motion of the perturbed model, Eqs. (8)-(12).

Note the efficient integration of general rotational motion is not a trivial matter and continuously motivates new research (see, for instance, Fukushima 2008, and references therein). In order to get an accurate solution, we decided to implement the chain of recurrences required by the Taylor method. We worked in quadruple precision and compute the Taylor series up to the order 33 to guarantee that the attained precision of numerical integration is far more than necessary for the comparison purpose with the predictions by our first-order analytical theory. The computation code was written in Fortran 77, compiled with the Pro Fortran Absoft compiler, and executed under Mac OS X. All the computations are conducted in a MacPro with two $3 \mathrm{GHz}$ dual-core Intel processors.

We found it convenient to use internal units in our computations. Thus, we took a mass unit of $8.11473 \times 10^{27} \mathrm{~kg}$, a length unit of one km, and a time unit of $472545.4 \mathrm{~s}$, which amounts to one Eulerian period of Ceres. In these units,

$$
\begin{array}{ll}
\epsilon=-1.48743 \times 10^{-7}, & n=0.020449, \\
a_{1}=97.1704, & a_{3}=90.8873 .
\end{array}
$$

We choose initial conditions $t=0, M_{0}=M(0)=1, \Lambda_{0}=$ $\Lambda(0)=\cos I_{0}, N(0)=\cos J_{0}, \mu_{0}=\mu(0)=0, \lambda_{0}=\lambda(0)=1$, and $v_{0}=v(0)=0$, and perform a short-term propagation of Hamilton equations, Eqs. (8)-(12). Figure 1 shows in the top plot that the modulus of the angular momentum is only affected 
Table 5. Coefficients of the periodic terms in the analytical solution for Ceres.

\begin{tabular}{|c|c|c|c|c|c|c|}
\hline \multirow[b]{2}{*}{ Frequency $(i, j)$} & \multirow[b]{2}{*}{ Period (days) } & \multicolumn{5}{|c|}{ Coefficient } \\
\hline & & $M / \epsilon$ & $\Lambda / \epsilon$ & $\mu-\delta$ & $v$ & $\lambda$ \\
\hline$(2,0)$ & 840.250 & - & $9.4943 \times 10^{+04}$ & $-1.0836 \times 10^{-05}$ & $-4.4828 \times 10^{-08}$ & $1.0896 \times 10^{-05}$ \\
\hline$(2,-1)$ & 0.35380 & $-7.6334 \times 10^{-02}$ & $-1.5267 \times 10^{-01}$ & $2.4028 \times 10^{-06}$ & $-2.4028 \times 10^{-06}$ & $-8.7482 \times 10^{-12}$ \\
\hline$(0,1)$ & 0.35365 & $7.6249 \times 10^{-02}$ & - & $2.4001 \times 10^{-06}$ & $-2.4001 \times 10^{-06}$ & $-8.7265 \times 10^{-12}$ \\
\hline$(2,1)$ & 0.35350 & $5.2298 \times 10^{-05}$ & $-1.0460 \times 10^{-04}$ & $1.6462 \times 10^{-09}$ & $-1.6462 \times 10^{-09}$ & $-1.8014 \times 10^{-14}$ \\
\hline$(2,-2)$ & 0.17686 & $-7.2861 \times 10^{-05}$ & $-7.2861 \times 10^{-05}$ & $2.2935 \times 10^{-09}$ & $-2.2935 \times 10^{-09}$ & $1.1475 \times 10^{-17}$ \\
\hline$(0,2)$ & 0.17683 & $-9.9901 \times 10^{-08}$ & - & $-3.1446 \times 10^{-12}$ & $3.1446 \times 10^{-12}$ & $1.1465 \times 10^{-17}$ \\
\hline$(2,2)$ & 0.17679 & $-3.4244 \times 10^{-11}$ & $3.4244 \times 10^{-11}$ & $-1.0779 \times 10^{-15}$ & $1.0779 \times 10^{-15}$ & $7.8652 \times 10^{-21}$ \\
\hline
\end{tabular}

Notes. The coefficients of $M$ and $\Lambda$ are scaled by $\epsilon=-5.40548 \times 10^{15} \mathrm{~kg} \mathrm{~m}^{2} / \mathrm{s}^{2}$ and are given in seconds; those of the angles are not scaled and are given in radians.
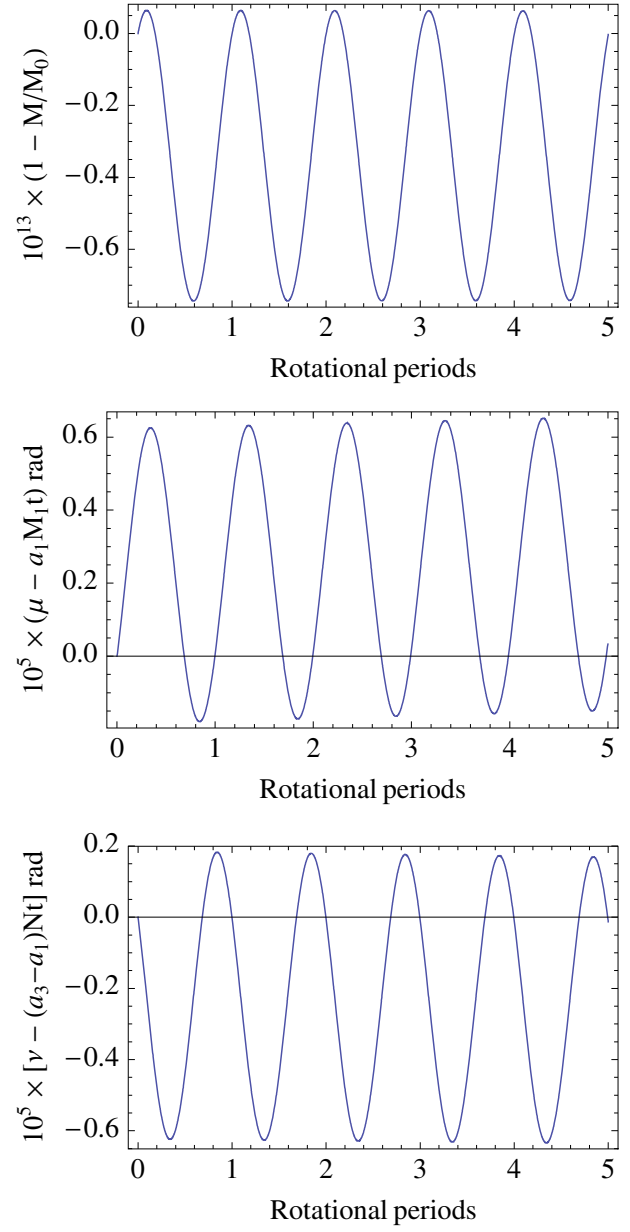

Fig. 1. Sample of Ceres' short-term propagation by the direct numerical integration of the equations of motion. The time scale is in units of the rotational period of the unperturbed motion.

by short period oscillations with the frequency of the unperturbed rotational period, Eq. (25), and very small amplitude. Similar oscillations happen to Andoyer's angles $\mu$ and $v$, as noted in the centre and bottom plots of Fig. 1. Finally, neither $\Lambda$ nor $\lambda$ are affected by appreciable short-term effects, and their shortterm propagation is not presented. Note that to better manifest the short-period effects in Andoyer's angles $\mu$ and $\nu$ in Fig. 1 we subtracted from each of them their linear, unperturbed part $a_{1} M t$ for $\mu$ and $-\left(a_{1}-a_{3}\right) N t$ for $v$. Besides, to enhance the short-term features, we present abscissas in terms of the torque-free rotation period.
Long-term features predicted by the analytical solution appear after a much longer propagation. Results are presented in Fig. 2, where the time evolution of $M$ shows that both shortand long-period effects have very low amplitudes. The plot in the second row of Fig. 2 shows that the short-period oscillations in $\mu$ are affected by a semiannual oscillation caused by the orbital motion of Ceres about the sun. Besides, we clearly note the secular drift, whose value agrees with the amplitude predicted in Eq. (29) because six Ceres' orbital periods amount to $\sim 0.276$ centuries.

Long-term features of $v$ are presented in the plot in the third row of Fig. 2. Even though the terms on the frequency $2 n$ are negligible in the analytical solution for $v$, the semiannual effect remains, although it is difficult to appreciate. It is a result of the almost equal amplitudes of terms in $(0,1)$ and $(2,-1)$, according to the values in Table 5, that cancel each other every $\pi / n$. Besides, the secular effects agree with the secular rate predicted by the theory as given in Eq. (27).

The last plots of Fig. 2 show that $\Lambda$, bottom plot, is free from secular terms, as predicted by the theory, and that the secular evolution of $\lambda$, plot in the fourth row agrees with the amplitude provided in Eq. (28).

After checking the qualitative agreement between the direct numerical integration and the analytical results, we investigate the quality of the analytical solution in quantitative terms. First of all, we compute the arbitrary integration constants of the theory for the initial conditions above. They are

$$
\begin{aligned}
& M_{1}=M_{0}+3.3973 \times 10^{-14} \\
& \Lambda_{1}=\Lambda_{0}-1.2437 \times 10^{-8} \\
& \mu_{1}=\mu_{0}-7.6648 \times 10^{-6} \\
& \nu_{1}=v_{0}-2.2292 \times 10^{-6} \\
& \lambda_{1}=\lambda_{0}+9.9076 \times 10^{-6}
\end{aligned}
$$

which are the values that must be replaced in Eqs. (13)-(17) and (20). Then, we evaluate these equations in a large set of points and compare their values with those obtained from the numerical integration of the Hamilton equations, Eqs. (8)-(12). Results are presented in Fig. 3, where the symbol $\Delta$ in ordinate labels means absolute error, and in Fig. 4, where $\delta$ means relative error. Note the periodic oscillation in the errors of $\Lambda$ and $\lambda$ are related only to semiannual effects, while short-period errors appear combined with long-period ones in the other variables. The growth of the absolute errors in $\mu$ and $v$ is related to the increase of the values of these angles, which may reach high values, yet the relative error maintains an acceptable accuracy as shown in Fig. 4. 

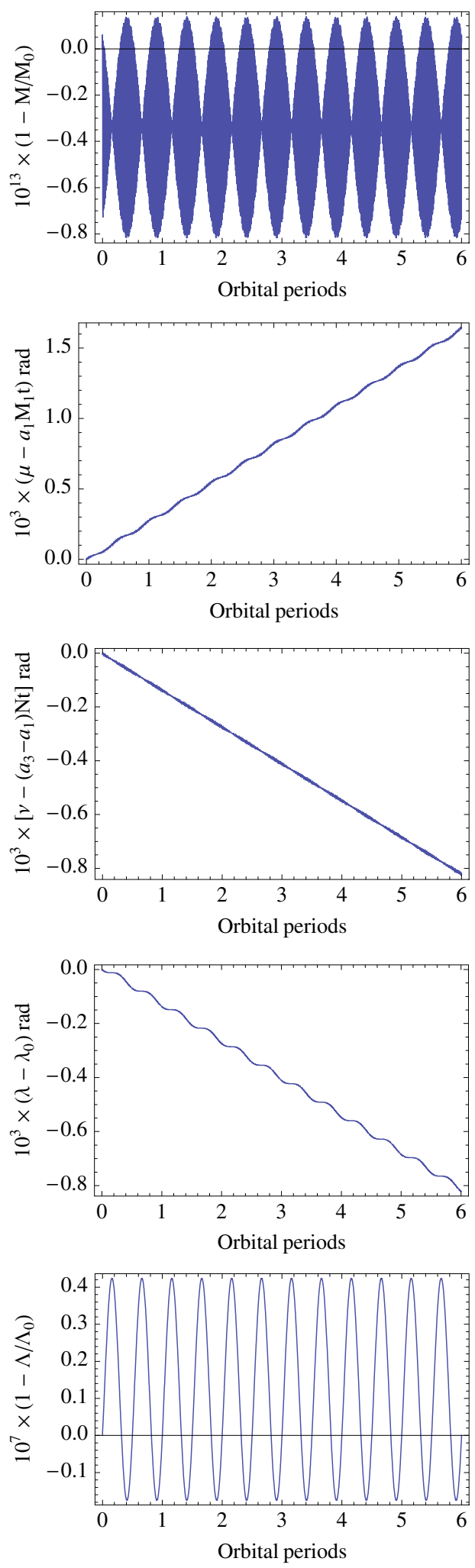

Fig. 2. Sample Ceres' long-term propagation by the direct numerical integration of the equations of motion. The time scale is in units of the orbital period of the perturber.

In order to illustrate the case of the critical inclinations we provide one more example, using Ceres' physical parameters above. It corresponds to the critical inclination of $54.7356 \mathrm{deg}$,
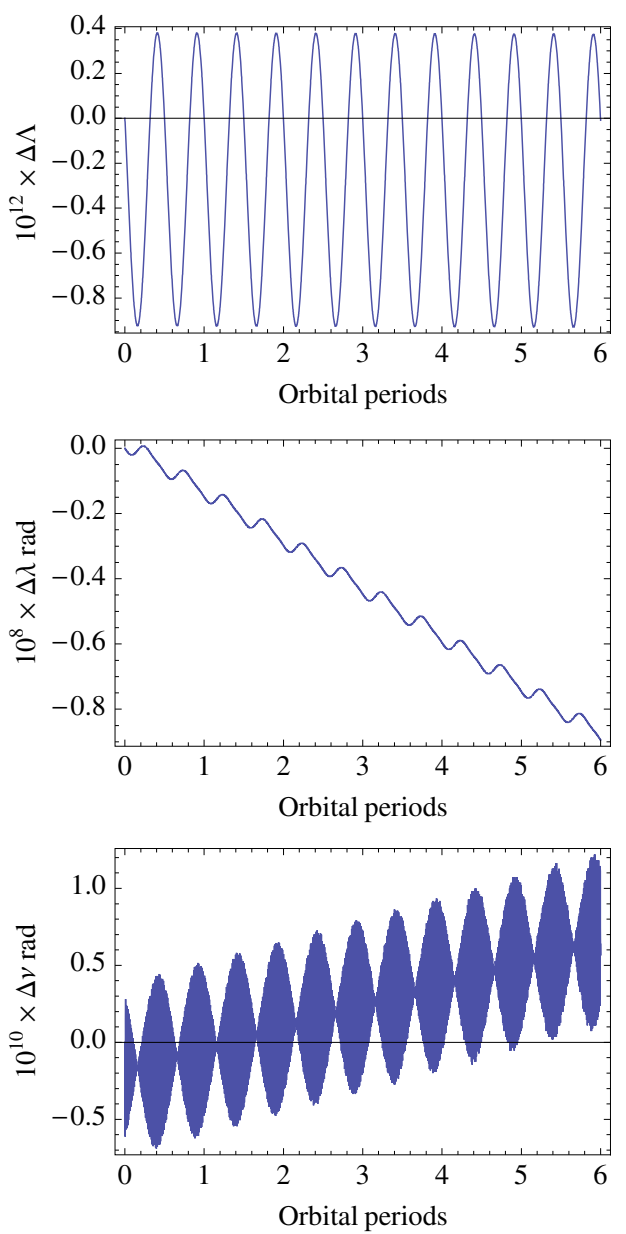

Fig. 3. Absolute errors of the analytical solution to the rotational motion of Ceres when compared with the numerical integration. The error evolution of $\mu$ is analogous to that of $\lambda$ and is not presented. The maximum error in $M$ remains bound to the last digit and is not shown.

for which we numerically integrate the equations of motion Eqs. (8)-(12) for the initial conditions: $t=0$ and

$$
\begin{array}{lll}
M_{0}=1, & \Lambda_{0}=\cos I_{0}=\frac{1}{\sqrt{3}}, & N_{0}=\cos J_{0}=\frac{1}{\sqrt{3}}, \\
\mu_{0}=0, & \lambda_{0}=1, & v_{0}=0 .
\end{array}
$$

As shown in Fig. 5, Andoyer variables evolve according to the pattern predicted by the first-order solution, with no secular perturbation effects in the rate of change of Andoyer angles except for a negligible drift in $\mu$, which after six orbital periods amounts to a few milli-arc seconds. A higher order secular effect is also noted in the motion of the node at a rate of few tenths of microarc second per orbital period.

We also check the accuracy of the first order solution for this case. Now we get for the initial conditions in Eq. (30)

$$
\begin{aligned}
& M_{1}=M_{0}+1.3600 \times 10^{-9}, \\
& \Lambda_{1}=\Lambda_{0}+1.8394 \times 10^{-9}, \\
& \mu_{1}=\mu_{0}+3.3071 \times 10^{-6}, \\
& \nu_{1}=v_{0}-5.7273 \times 10^{-6} \\
& \lambda_{1}=\lambda_{0}-6.9553 \times 10^{-10}
\end{aligned}
$$

The absolute errors in the propagation of the first-order solution are presented in Fig. 6. Errors in $v$ are not presented because the first-order solution always reaches the numerical precision. 
M. Lara et al.: Rotation of an oblate rigid body under external torque
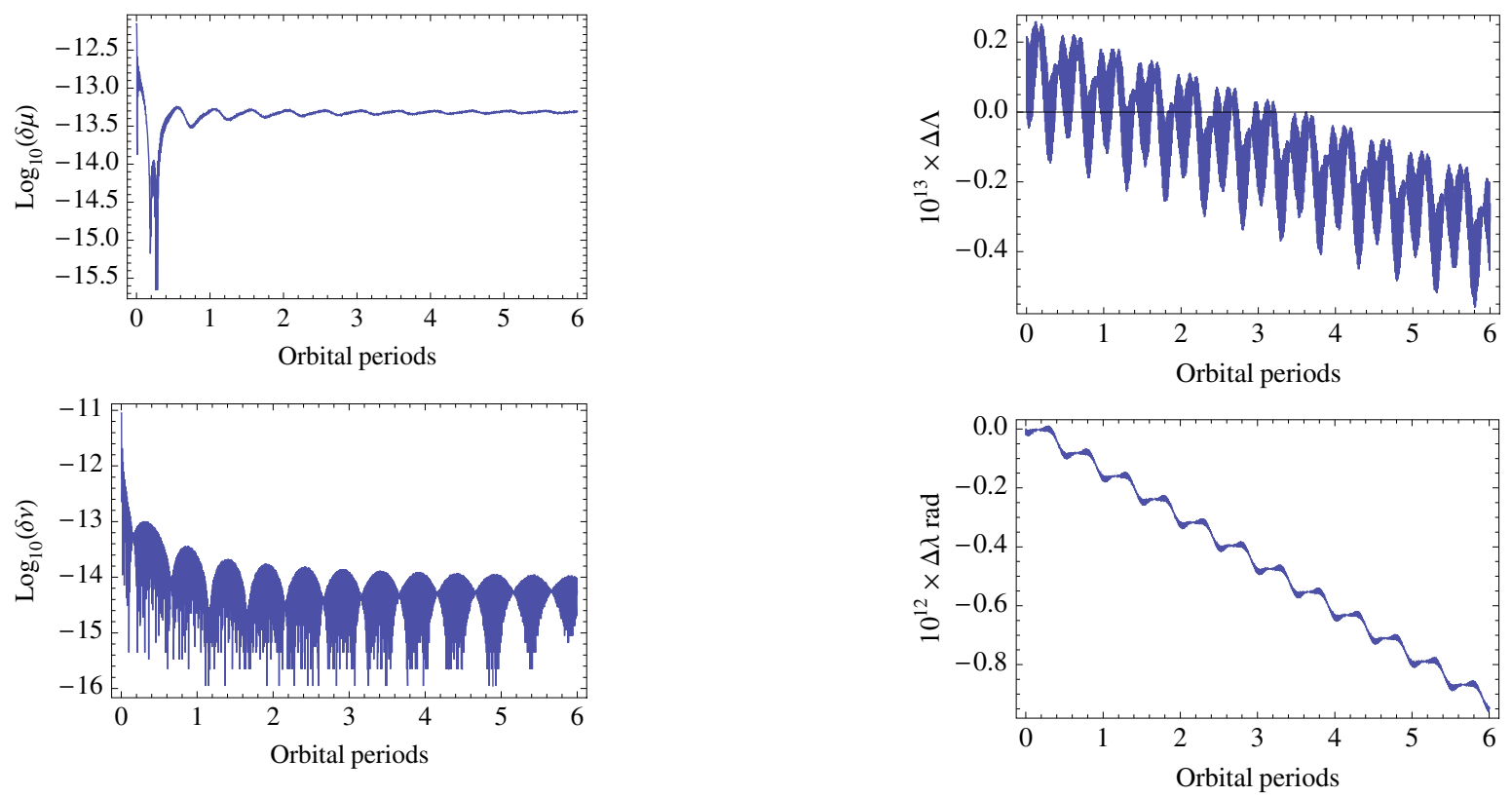

Fig. 4. Relative errors of the analytical solution to the rotational motion of Ceres when compared to the direct numerical integration.
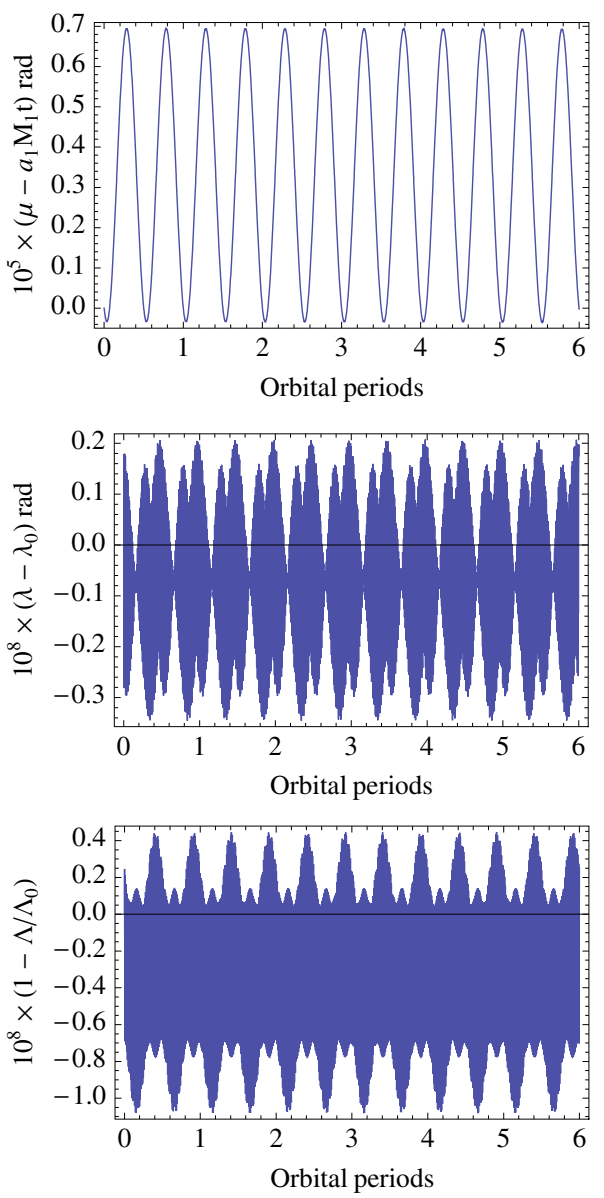

Fig. 5. Sample of the long-term propagation for the critical inclination of 54.7356 deg using Ceres' physical parameters. The evolution of $v$ and $M$ are analogous to that of $\mu$ and $\Lambda$, respectively, and are not presented. The time scale is in units of the orbital period of the perturber.

We note in Fig. 6 that the error in $\mu$ seems to grow in a quadratic way - although, as shown in Fig. 7, the accuracy of analytical propagation is very good, remaining with more than 13

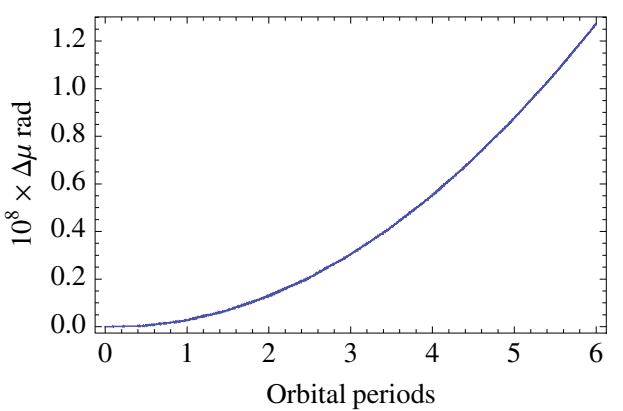

Fig. 6. Accuracy of the first-order solution for the critical inclination of 54.7356 deg using Ceres' physical parameters when compared to the direct numerical integration. The error in $M$ evolves similarly to that of $\Lambda$ and is not presented.

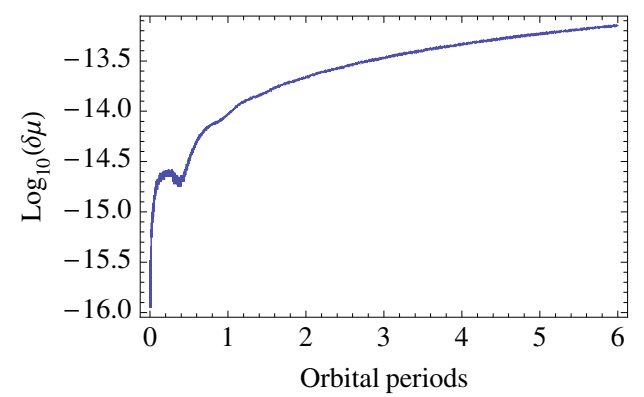

Fig. 7. Relative error of the first-order solution for $\mu$ for the critical inclination of 54.7356 deg using Ceres' physical parameters when compared to the direct numerical integration.

exact digits throughout this long period. Anyway, the magnitude of the error is consistent with the secular drift observed in the numerical propagation. A higher order theory should demonstrate that the higher order terms in the solution prevent rigid bodies at this inclination from evolving as unperturbed in the long term.

Similar results with respect to either the numerical integration or the accuracy of the first order solution have been found for the other special configurations. 


\section{Conclusions}

Simple assumptions in perturbed rigid body motion are enough to reveal the main characteristics of the rotational dynamics of a variety of celestial bodies. Without need of resorting to series developments, the perturbed motion is represented in closed form with convenient equations in which first-order effects are clearly identified. The relevant frequencies introduced by the perturbation are related to semiannual, diurnal and semidiurnal effects.

The computation of the theory was facilitated by using Andoyer variables, which show that the model considered evolves as unperturbed, in the long term, at five special configurations related to critical inclinations of the equatorial plane of the body and the orbital plane of the perturber with respect to Andoyer's plane. Andoyer variables suffer from known singularities. However, for singular Andoyer variables one may adopt alternative variables, as found in the literature.

The first-order solution is general, under the rough assumptions of this paper, but it is specifically well suited for the dwarf-planet Ceres, where the solution may be further simplified and provides an accuracy of the order of few milli-arc seconds that seems sufficient for the actual observational accuracy. However, in view of the ongoing NASA DAWN mission, which will visit Vesta and Ceres in the near future, a specific theory for ephemeris computation of the rotational motion of Ceres and Vesta is desirable. It would require increasing the precision of the theory by computing a higher order, and also by considering the eccentricity of the orbit about the sun.

Acknowledgements. We acknowledge comments of A. Escapa, University of Alicante, on an early version of the manuscript. Comments of the referee drew our attention to relevant literature and motivated us to improve the periodic terms of the solution in this paper. Support is acknowledged from a grant "Fundación Séneca" of the autonomous region of Murcia, and projects ESP 2007-64068 and AYA 2009-11896 (M.L.), and MTM 2009-10767 (S.F.) of the Goverment of Spain.

\section{References}

Andoyer, M. H. 1923, Cours de Mécanique Céleste (Paris: Gauthier-Villars et cie)

Barkin, Yu. V. 1980, Kosm. Issled., 18, 191 (Cosmic Res., 18, 267)

Barkin, Yu. V., \& Ferrándiz, J. M. 2005, Astron. Astroph. Trans., 24, 61

Beletskii, V. V. 1966, Motion o fan artificial satellite about the center of mass, Israel Prog. for Scientific Trans., Jerusalem

Bois, E. 1986, Celest. Mech., 39, 309

Bouquillon, S., Kinoshita, H., \& Souchay, J. 2003, Celest. Mech. Dyn. Astron., 86,29

Campbell, J. A., \& Jefferys, W. H., 1970, Celest. Mech., 2, 467

Carry, B., Dumas, C., Fulchignoni, M., et al. 2008, A\&A, 478, 235

Chernous'ko, F. L. 1963, PMM, 27, 474 (JAMM 27, 708)

Chierchia, L., \& Gallavotti, G. 1994, Annales IHP, 60, 1

Crenshaw, J. W., \& Fitzpatrick, P. M. 1968, AIAA J., 6, 2140

Deprit, A. 1967, Am. J. Phys., 35, 424

Deprit, A. 1969, Celest. Mech., 1, 12

Drummond, J., \& Christou, J. 2008, Icarus, 197, 480

Escapa, A., Getino, J., \& Ferrándiz, J. M. 2002, A\&A, 389, 1047

Fukushima, T. 1994, Celest. Mech. Dyn. Astron., 60, 57

Fukushima, T. 2008, AJ, 135, 2298

Getino, J., \& Ferrándiz, J. M. 1991, Celest. Mech. Dyn. Astron., 51, 35

Getino, J., \& Ferrándiz, J. M. 2001, MNRAS, 322, 785

Henrard, J. 2005, Icarus, 178, 144

Holland, R. L., \& Sperling, H. J. 1969, AJ, 74, 3

Hori, G.-i. 1966, PASJ, 18, 287

Kinoshita, H. 1972, PASJ, 24, 423

Kinoshita, H. 1977, Celest. Mech., 15, 277

MacCullagh, J. 1840, Proc. R. Irish Acad., 2, 520

Markeev, A. P. 1985, Kosm. Issled., 23, 323 (Cosmic Res., 23 267)

Mastropietro, V. 2001, Regular Chaotic Dyn., 6, 355

Mathews, P. M., Herring, T. A., \& Buffett, B. A. 2002, JGR (Solid Earth), 107, 2068

Michalak, G. 2000, A\&A, 360, 363

Moons, M. 1982, Celest. Mech., 26, 131

Morbidelli, A., Gonczi, R., Froeschlé, Ch., et al. 1994, A\&A, 282, 955

Russell, C. T., Coradini, A., Christensen, U., et al. 2003, Planet. Space Sci., 52, 465

Scheeres, D. J. 2009, Celest. Mech. Dyn. Astron., 104, 103

Shirai, T., \& Fukushima, T. 2000, AJ, 119, 2475

Souchay, J., Loysel, B., Kinoshita, H., \& Folgueira, M. 1999, A\&AS, 135, 111

Thomas, P. C., Parker, J. W., McFadden, L. A., et al. 2005, Nature, 437, 224 